\title{
Discovery of Solar Rieger periodicities in another star
}

\author{
M. Massi, J. Neidhöfer, Y. Carpentier, and E. Ros \\ Max-Planck-Institut für Radioastronomie, Auf dem Hügel 69, 53121 Bonn, Germany \\ e-mail: [mmassi; jneidhoefer;ros]@mpifr-bonn.mpg.de; yvain2020@yahoo.fr \\ Received 13 September 2004 / Accepted 6 March 2005

\begin{abstract}
The Rieger periods are solar cycles with a time scale of months, which are present in both flaring activity and sunspot occurrence. These short-term periodicities, tentatively explained by equatorially trapped Rossby-type waves modulating the emergence of magnetic flux at the surface, are considered a peculiar and not yet fully understood solar phenomenon. We chose a stellar system with solar characteristics, UX Arietis, and performed a timing analysis of two 9-year datasets of radio and optical observations. The analysis reveals a 294-day cycle. When the two 9-year datasets are folded with this period, a synchronization of the peak of the optical light curve (i.e., the minimum spot coverage) with the minimum radio flaring activity is observed. This close relationship between two completly independent curves makes it very likely that the 294-day cycle is real. We conclude that the process invoked for the Sun of a periodical emergence of magnetic flux may also be applied to UX Arietis and can explain the cyclic flaring activity triggered by interactions between successive cyclic emergences of magnetic flux.
\end{abstract}

Key words. stars: individual: UX Arietis - radio continuum: stars - stars: flare - Sun: oscillations

\section{Introduction}

RS CVn stars are tightly-orbiting binary systems, consisting of an $\mathrm{F}$ or $\mathrm{G}$ type dwarf and a $\mathrm{G}$ or $\mathrm{K}$ type subgiant. The components of these systems are fast rotators due to the strong tidal interaction that synchronizes stellar spins and orbital motion, making the dynamo mechanism very effective: Quite extended (covering up to several percent of the stellar surface) cool spots on the more active star of the system and intense X-rays and radio flares have been observed (Hall 1976; Owen et al. 1976; Budding et al. 2002). Spots and flares are related to each other: Solar and stellar spots are created by intense magnetic fields pushing their way through the photosphere and as observed on the Sun, flares are triggered by interactions between new and older emergences of magnetic flux in the same area (Nishio et al. 1997).

One of the most active RS CVn systems is UX Arietis (Beasley \& Güdel 2000). The flaring activity of UX Arietis shows some periodicities: 3 -yr monitoring with the Effelsberg $100-\mathrm{m}$ radio telescope revealed a cycle of several months modulated by shorter cycles (Massi et al. 1998) and later 3-yr radio monitoring with the Green Bank Interferometer not only confirmed the existence of such a cyclic flaring activity in UX Arietis, but also indicated periodical activity in other stars (Richards et al. 2003).

Where do these periodicities come from? If spots and flares are related to each other (like in the Sun), does a periodical flaring activity imply a similar periodicity in the spot occurrence? The best known periodicity in the production of energetic flares is the Solar Rieger periodicity of 152-158 days (Rieger et al. 1984; Bai 2003). Oliver et al. (1998) have examined temporally coincident datasets and discovered that an identical periodicity is present in the occurrence of sunspots.

Our research on short-term stellar periodicities (Massi et al. 1998; Massi et al. 2002) is here extended to adress the following question: does the periodical radio flaring activity in UX Arietis imply a periodical spot occurrence? With this aim we have performed a timing analysis on two 9-year datasets of radio and optical observations. Section 2 describes the two data bases, Sect. 3 the timing analysis, and finally, Sect. 4 presents our conclusions.

\section{The data}

The Effelsberg 100-m-telescope observed UX Arietis over a frequency range 1.4-43 GHz for almost 3400 days with quite irregular sampling. The largest gap in the Effelsberg data base is filled by the Green Bank Interferometer (GBI) data base at 2.25/8.3 GHz with at least 2 observations per day. Figure 1d shows the radio observations and Table 1 gives their relative time schedules. A visual inspection of Fig. 1d shows several large flares ( $S \geq 250 \mathrm{mJy}$ ). Massi et al. (1998) analyzed the data set (JD $2448965.17+$ ) $0 \div 1000$ and determined periods of 14, 25, 159 and 56 (circular polarization data) days. Richards et al. (2003) analyzing the GBI data identified periods of 53, 83,141 and 280-300 days. This is very similar to the Sun, where together with the Rieger period (152-158 days) several other periodicities with time scales of months appear and disappear and slightly change in frequency (see references in Oliver \& Ballester 1995; Bai 2003). Here, we analyze the full available radio data set, i.e., the above mentioned Effelsberg 

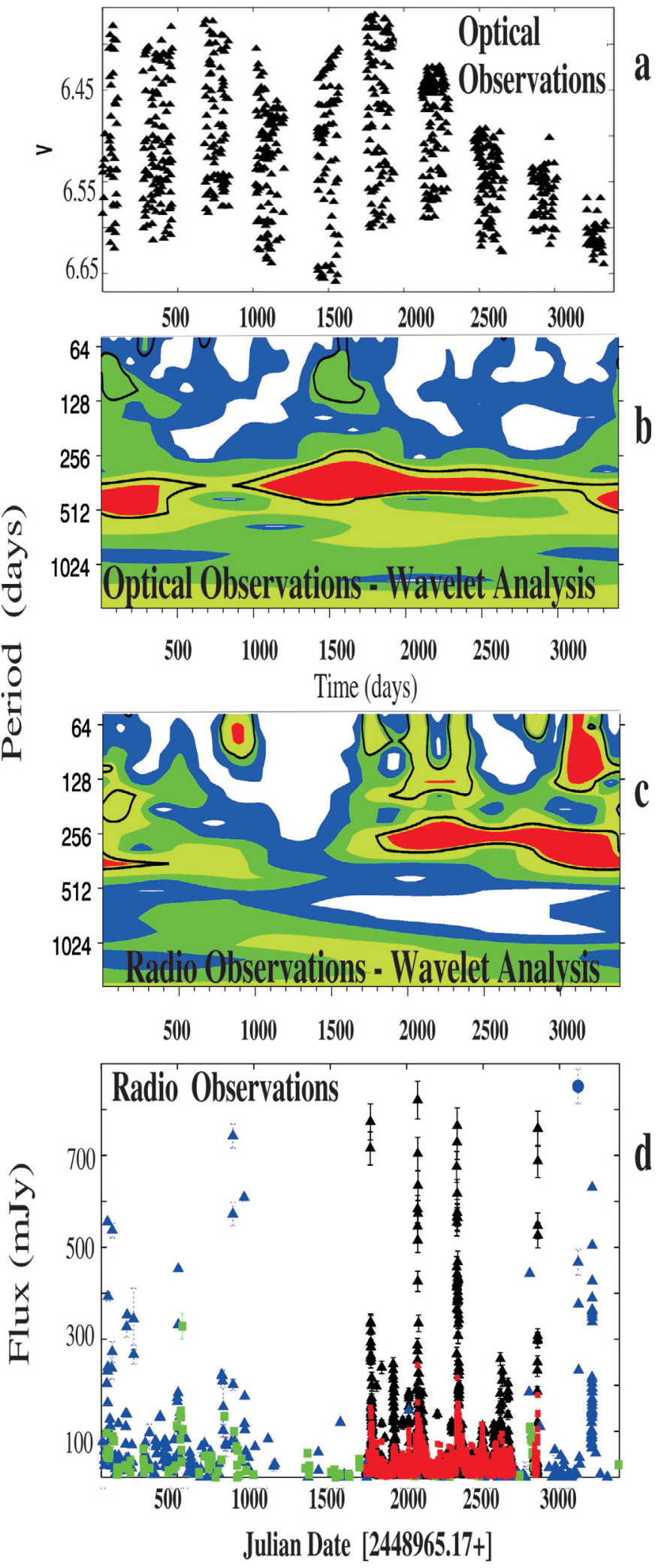

Fig. 1. Radio and optical measurements and their corresponding wavelet spectra. The $x$-axis of all figures is the same and equal to the Julian Date [JD-2 448 965.17]. Figures a) and d) are described in Sect. 2; Figs. b) and c) in Sect. 3. The radio observations d) are: Effelsberg data above $8 \mathrm{GHz}$ (blue triangles; the blue dot is an out of scale flare of $1318 \mathrm{mJy}$ ), and below $8 \mathrm{GHz}$ (green squares), Green Bank Interferometer data at $8.3 \mathrm{GHz}$ (black triangles), and at $2.25 \mathrm{GHz}$ (red squares). 100-m telescope data and the GBI monitoring and further new Effelsberg data (see Table 1). The photometric observations of UX Arietis used here are data taken with the T3 0.4-metre Automatic Photoelectric Telescope at Fairborn Observatory by Aarum Ulvås \& Henry (2003) and consist of 869 V band measurements over 9 years (see Fig. 1a and Table 1).

\section{Methods of timing analysis: Wavelets and PDM}

Wavelet analysis decomposes a one-dimensional time series into a two-dimensional time-frequency space and displays the power spectrum in a two dimensional color-plot presenting how the Fourier periods $(y)$ vary in time $(x)$ (Torrence \& Compo 1998). The Morlet wavelet results are presented in Fig. $1 b$ for the optical data and in Fig. 1c for the radio data. The power of the frequency component is in arbitrary units (red stands for the dominant one). The black contour lines give the $90 \%$ confidence level obtained by assuming a red noise background spectrum (Torrence \& Compo 1998). Dominant periodicities for both radio and optical data are evident in the range 256-512 days (red area). In addition, the radio data show power at shorter time scales (i.e. $<256$ days). These are the periodicities discussed in Massi et al. (1998) and Richards et al. (2003). In the optical data these shorter periodicities are significant (within black contour lines) but with little power, therefore, they will not be taken into account in the following discussion which focuses on the periodicities occurring in the interval of 256-512 days.

The data have been further analyzed with the Phase Dispersion Minimization method (PDM, Stellingwerf 1997), which is one of the most efficient methods for use with irregularly spaced data. The results are plotted in Fig. 2. The analysis shows that the two radio periods, $P_{\text {radio }_{1}}=288 \pm 6$ days and $P_{\text {radio }_{2}}=383 \pm 9$ days, both have an optical counterpart within the respective errors, i.e., $P_{\mathrm{opt}_{1}}=301 \pm 7$ days and $P_{\mathrm{opt}_{2}}=386 \pm 11$ days. The influence of noise in the power spectrum and the estimate of the significance of the determined peaks have been established using the method of Fisher randomization (Nemec \& Nemec 1985), implemented in the Starlink software package PERIOD. This resulted in false alarm probabilities below 0.01 with $95 \%$ confidence for all four periods. However, due to gaps in the sampling of the optical data, we discard $P_{2}$. This is because if the optical data are folded with $P_{\mathrm{opt}_{2}}$, there is a lack of data over a consistent phase interval resulting in an artificial minimum (see the regular holes in sampling in Fig. 1a). This is completely different in the case of the first period of 288-301 days. Figure 3 presents the data folded with a period of $P=294$ days, which is the best common period for both data sets (radio and optical data). First, all phase intervals (see Fig. 3) are filled with data and no obvious lack of data is present in the light curves. Second, optical data (Fig. 3 top) show a maximum (i.e., a minimum spot coverage) at phase $\Phi=0.4$ exactly where the radio data (Fig. 3 bottom) show a minimum (i.e. minimum in flaring activity). 
Table 1. Log of radio and optical observations. Start and Stop are given in Julian Date JD-2 448965.17 (1992 Dec. 8).

\begin{tabular}{ccccc}
\hline \hline & Time interval & Telescope & Data points & References \\
\hline Radio & $0 \div 1000$ & Effelsberg 100-m & 230 & Massi et al. (1998) \\
& $1000 \div 3386.95$ & Effelsberg 100-m & 211 & This work \\
& $1732.71 \div 2858.80$ & Green Bank Interferometer & 2533 & Richards et al. (2003) \\
Optical & $1.59 \div 3393.46$ & Fairborn Automatic Photoelectric Telescope & 869 & Aarum Ulvås \& Henry (2003) \\
\hline
\end{tabular}
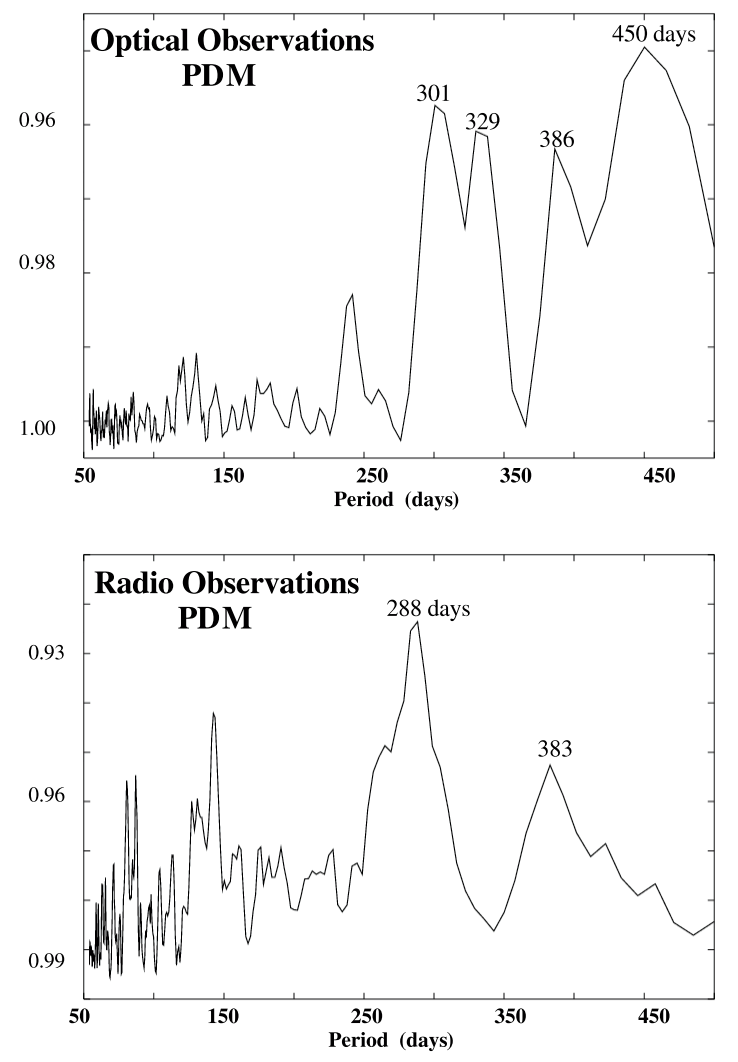

Fig. 2. Result of the Phase Dispersion Minimization analysis. Note that the analysis provides the most probable period as a minimum (Stellingwerf 1997) and the vertical scale is shown reversed here.

\section{Discussion and conclusions}

The results of our timing analysis of a data base of radio measurements (Fig. 1d) and a temporally coincident data base of photometric $V$ observations (Fig. 1a) of UX Arietis, are:

1. The Wavelet analysis over a total time interval of 3400 days shows the presence of a quasi-periodic oscillation in the range 256-512 days in both optical and radio data (Figs. $1 \mathrm{~b}$ and c).

2. The PDM analysis of the radio data determines that the quasi-periodic oscillation between 256-512 days consists of two distinct periods: $P_{\text {radio }_{1}}=288 \pm 6$ days (the dominant one) and $P_{\text {radio }_{2}}=383 \pm 9$ days (Fig. 2). The PDM analysis of the optical data determines a period $P_{\mathrm{opt}_{1}}=301 \pm$ 7 days, coincident within the errors with the radio periodicity $P_{\text {radio }_{1}}$, and also a period $P_{\mathrm{opt}_{2}}=386 \pm 11$ days, coincident with the radio-periodicity $P_{\mathrm{radio}_{2}}$. Therefore, both
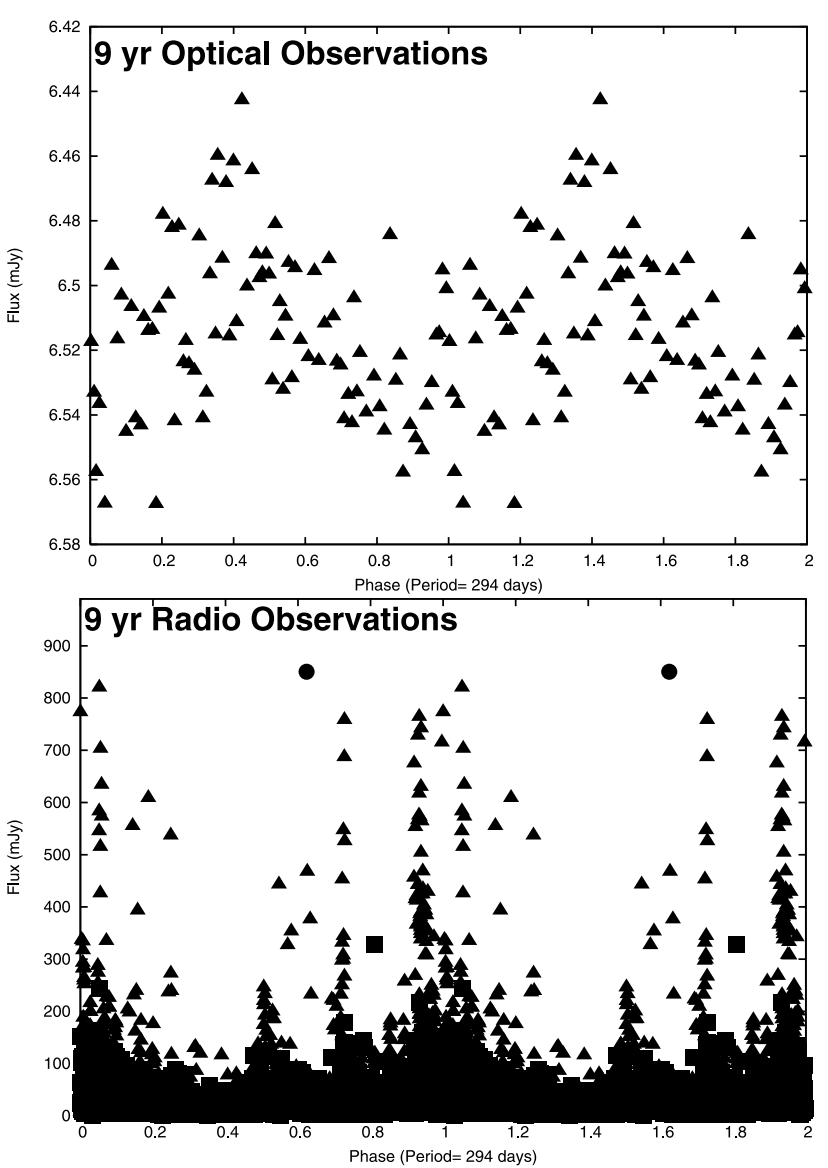

Fig. 3. Bottom: folding of the radio data with a period of 294 days. Phase 0 refers to $t_{0}$ as in Table 1 . The phase interval 0-1 is repeated twice. There is a clear lack of energetic flares at $\Phi=0.4$. Top: folding of the photometric $V$ data with the same period of 294 days, as the radio data. The data are averaged over phase bins of 0.01 . The peak for $V$ at $\Phi=0.4$ corresponds to a minimum in spot coverage and is well synchronized with the "hole" of radio flaring activity.

periods of the radio flaring activity have corresponding optical counterparts.

3. The analysis of the data folded with the two determined periods confirms and corroborates $P_{1}$ whereas $P_{2}$ appears to be biased by the gaps in the sampling. When the data are folded in phase with $P=294$ days (the best common, optical and radio, period for $P_{1}$ ) show a good phase coverage (Fig. 3). Moreover, there exists a relationship between the radio curve and the optical curve: the maximum $V$ magnitude (i.e. the minimum spot coverage) is synchronized with the minimum in radio flaring activity. Maximum $V$ magnitude and minimum radio flux density occur both at 
phase $\Phi=0.4$ (Fig. 3). This close relationship between two completly independent curves makes it very likely that the 294-day cycle is real.

On the basis of these results we conclude that a periodicity seems to be present on UX Arietis. This 294-day cycle cannot be an artifact related to a possible spot migration. In fact, it would imply an orbital phase migration rate of $1.24 \mathrm{yr}^{-1}$ whereas a maximum value of $0.26 \mathrm{yr}^{-1}$ has been measured (Aarum Ulvås \& Henry 2003). We are therefore faced with a real intrinsic stellar periodicity.

As already mentioned in the previous sections, periodicities with a time scale of months are well established on the Sun; they are called "Rieger periodicities" from the name of their discoverer (Rieger et al. 1984). They have been observed in $\gamma$-ray, X-ray (Rieger et al. 1984) and $\mathrm{H} \alpha$ flares (Ichimoto et al. 1985), in flares at radio wavelengths (Bogart \& Bai 1985), in daily sunspot areas (Oliver et al. 1998) and numbers (Ballester et al. 1999) and even in variations of the solar neutrino flux (Sturrock et al. 1999).

It has been suggested that Rieger periodicities in solar neutrino data may be due to $r$-modes (Rossby waves) moving magnetic regions in and out of the path of neutrinos propagating from the solar core to the Earth, assuming an influence of the magnetic field on the propagation of the neutrinos (Sturrock 2003) possibly by the resonant spin flavor precession mechanism ( Akhmedov 1997; Pulido \& Akhmedov 2000). Moreover, and of interest for the flare production considered here, equatorially trapped Rossby-type waves might modulate the emergence of magnetic flux (Lou 2000). In this respect (Ballester et al. 1999), periodic emergence of magnetic flux through the photosphere may operate in two different ways, either forming spots within already established active regions (and therefore inducing flares by magnetic reconnection) or by forming new spots away from active regions. Our result (the same period of 294 days in temporal coincidence for optical and radio data) is in good agreement with the first of the two cases, i.e. the periodical emergence of magnetic flux occurs in a defined geometrically localized area. The suggested scenario is therefore as follows: during the initial phase (of the 294-day cycle) the spotted surface progressively increases because of the emersion of new magnetic structures. The emergence area however remains roughly localized (Ballester et al. 1999; Lanza et al. 2001), so that new and older magnetic structures interact with each other and large $(S \geq 250 \mathrm{mJy})$ flares can be observed ( $\Phi$ in the range $0.6 \div 1.2$ in Fig. 3-bottom). Then the minimum of the cycle follows, i.e the emersion of magnetic flux decreases or stops. Because there are no new spots and the the older spots dissipated, the optical light curve has its maximum $(\Phi=0.4$ in Fig. 3 top). The radio flaring activity dramatically drops and the radio curve shows a clear "hole" or lack of flaring activity, exactly at the same phase $(\Phi=0.4)$.
Acknowledgements. We wish to thank Karl M. Menten, Matthias Kadler and Edward Polehampton for their careful reading of the manuscript and their comments. The results are based on observations with the 100-m telescope of the Max-Planck-Institut für Radioastronomie at Effelsberg. The Green Bank Interferometer is a facility of the National Science Foundation operated by the NRAO in support of NASA High Energy Astrophysics programs. This research made use of the data provided by V. Aarum Ulvås to the SIMBAD database, operated at CDS, Strasbourg, France. Wavelet software was provided by C. Torrence and G. P. Compo, and available at URL: http://paos.colorado.edu/research/wavelets/. We acknowledge the data analysis facilities provided by the Starlink Project which is run by CCLRC on behalf of PPARC.

\section{References}

Aarum Ulvås, V., \& Henry, G. W. 2003, A\&A, 402, 1033

Akhmedov, E. Kh. 1997, Proc. 4th International Solar Neutrino Conference, Heidelberg, Germany [hep-ph/9705451]

Bai, T. 2003, ApJ, 591, 406

Ballester, J. L., Oliver, R., \& Baudin, F. 1999, ApJ, 522, L153

Beasley, A. J., \& Güdel, M. 2000, ApJ, 529, 961

Bogart, R. S., \& Bai, T. 1985, ApJ, 299, L51

Budding, E., Lim, J., Slee, O. B., \& White, S. M. 2002, New Astron., $7,1,35$

Hall, D. 1976, in Multiple Periodic Variable Stars, ed. W. Fitch (Dordrecht: Reidel), IAU Coll., 29, 287

Ichimoto, K., Kubota, J., Suzuki, M., Tohmura, I., \& Kurokawa, H. 1985, Nature, 316, 422

Lanza, A. F., Rodonò, M., Mazzola, L., \& Messina, S. 2001, A\&A, 376, 1011

Lou, Y. Q. 2000, ApJ, 540, 1102

Massi, M., Neidhöfer, J., Torricelli-Ciamponi, G., \& Chiuderi-Drago, F. 1998, A\&A, 332, 194

Massi, M., Menten, K., \& Neidhöfer, J. 2002, A\&A, 382, 152

Nemec, A. F., \& Nemec, J. M. 1985, AJ, 90, 2317

Nishio, M., Yaji, K., Kosugi, T., Nakajima, H., \& Sakurai, T. 1997, ApJ, 489, 976

Oliver, R., \& Ballester, J. L. 1995, Sol. Phys., 156, 145

Oliver, R., Ballester, J. L., \& Baudin, F. 1998, Nature, 394, 552

Owen, F. N., Jones, T. W., \& Gibson, D. M. 1976, ApJ, 210, L27

Pulido, J., \& Akhmedov, E. Kh. 2000, Astroparticle Phys., 13, 227

Richards, M. T., Waltman, E. B., Ghigo, F. D., \& Richards, D. St. P. 2003, ApJS, 147, 337

Rieger, E., Kanbach, G., Reppin, C., et al. 1984, Nature, 312, 623

Stellingwerf, R. F. 1997, ApJ, 486, 886

Sturrock, P. A., Scargle, J. D., Walther, G., \& Wheatland, M. S. 1999, ApJ, 523, L177

Sturrock, P. A. 2003, Am. Astron. Soc. Solar Physics Division Meeting 34.08.05

Torrence, C., \& Compo, G. P. 1998, Bull. Am. Meteo. Soc., 79, 61 